\title{
COORDINATION ON EGALITARIAN NETWORKS FROM ASYMMETRIC RELATIONS IN A SOCIAL GAME OF CHICKEN
}

\author{
MILENA TSVETKOVA \\ Department of Sociology, Cornell University \\ 372 Uris Hall, Ithaca, New York 14853, USA \\ mvt9@cornell.edu \\ VINCENT BUSKENS \\ Department of Sociology/ICS, Utrecht University \\ Padualaan 14, Utrecht, 3584 CH, Netherlands \\ v.buskens@uu.nl
}

Received 10 August 2012

Revised 15 November 2012

Accepted 26 December 2012

Published 7 March 2013

\begin{abstract}
We present a model of social interaction in which actors choose their partners and play the Chicken Game with them. In contrast to most previous models of the coevolution of games and networks, we assume that the actors can employ different actions against different partners. This allows us to derive two different solutions to the coordination and asymmetry problems in the game: egalitarian stationary conventions (based on indirect reciprocity at the network level) and alternating conventions (based on direct reciprocity in dyads). We derive predictions on the occurrence of the possible convention structures and test our theoretical findings in a computerized experiment. The experimental results indicate that the egalitarian conventions indeed emerge and persist and that the alternating conventions are much more likely than the stationary ones. However, although egalitarian in terms of payoffs, the convention structures tend to have hierarchical action distributions.
\end{abstract}

Keywords: Game theory; experimental sociology; social networks; reciprocity.

PACS Number(s): 89.65.-s, 02.50.Le

\section{Introduction}

There is a new and rapidly developing research field that studies the coevolution of behavior and social structure with models of "social games" (also known as adaptive coevolutionary networks [22]). In such models, individuals have to choose partners in a network game, as well as an action in a standard two-player two-action game [42, 46]. These games are essentially multi-player versions of an older generation of models of games with an exit option $[43,49]$. A number of analytical, simulation, and experimental studies exist for social games of cooperation and coordination based on the Prisoner's Dilemma [14, 23, 41, 50], the Coordination Game [10, 12, 
$19,26,27]$, and the games of Stag-Hunt [11, 44] and Chicken $[5,6,9]$. With a few exceptions $[13,16,24,25]$, most of these studies assume that actors choose the same action against all of their partners. For example, if an actor cooperates with one partner in a cooperation game, she also has to cooperate with all her other partners at that time point. Thus, these models focus on actor states or types and are applicable to individual choices that are hard to "customize" per interaction, such as espousing a political perspective, specializing in a profession, or acquiring a new communication technology. This kind of individual behavior is associated with mechanisms such as imitation, influence, and homophily and with network phenomena such as diffusion cascades, assortativity, and segregation.

However, in everyday social life, we are often involved in directed, person-specific interactions, relations, and exchanges such as calling a relative on the phone, being a subordinate to the boss, giving advice to a friend, doing a favor to a neighbor, lending a hand to a stranger. This behavior is related to mechanisms such as interpersonal comparison, reciprocity, prestige, and exclusion. These mechanisms are rarely associated with network effects and this is why coevolution models have ignored partner-specific behavior. In the current paper, we overcome this research bias and investigate theoretically and experimentally how asymmetric partner-specific interactions can affect network structure.

We present a model and an experimental test of a social game in which actors choose partners and play the Chicken Game with them. The dilemma in the game is that the sum of payoffs is maximized only when one of the partners receives more than the other. The actors can resolve this dilemma and achieve an egalitarian outcome by coordinating on one of two different kinds of conventions - an alternating convention based on direct reciprocity $[1,2,47]$ or an egalitarian stationary convention based on indirect reciprocity [8, 36, 37] (also known as generalized reciprocity or generalized exchange [33]). In an alternating convention, actors prevent unequal exchange by taking turns to "give" to each other. In an egalitarian stationary convention, all the actors remain equally well-off by balancing taking from half of their partners with giving to the other half. The first convention occurs at the level of dyads and has been previously observed in experiments [7, 40]. We present the second convention as a theoretical network-level solution to the problem of producing egalitarian macro-level outcomes from asymmetric dyadic relations. We investigate the different network structures that the two types of conventions lead to and how often these are expected to occur. In addition, we use a laboratory experiment to test whether small groups of individuals are more likely to coordinate on the dyadic solution compared to the network solution and whether certain convention structures are more common than others.

In terms of the social problem it addresses, our study contributes to a long line of research that explains the importance of reciprocity for increasing collective welfare. By focusing on the Prisoner's Dilemma, this literature has repeatedly demonstrated the importance of reciprocity for efficiency $[1,47]$. The questions of whether and how 
reciprocity can bring about and sustain equality, however, have been largely ignored. Our study attempts to fill this gap by using the Chicken Game to investigate the roles of direct and indirect reciprocity in bringing about egalitarian macrolevel outcomes from asymmetric dyadic relations. Such relations form the basis of mutual aid and cooperation in human societies but also provide a mechanism for the emergence of inequalities and hierarchies. A better understanding of the extent to which direct and indirect reciprocity succeed in preventing and/or eradicating inequalities can inform strategies for managing polarization and conflict in social groups.

\section{Formal Model and Analytical Solutions}

\subsection{Social game}

The social game has two components: the network game in which actors choose their partners and the underlying Chicken Game played by each pair of connected actors. Each actor's strategy in the social game is determined by the combination of her strategies in the two constituent games.

Formally, consider a finite set of actors $N=\{1,2, \ldots, n\}$ who can choose partners to interact with. Let $l_{i}=\left(l_{i 1}, \ldots, l_{i(i-1)}, l_{i(i+1)}, \ldots, l_{i n}\right)$ be the vector of link decisions of actor $i$, where $l_{i j} \in\{0,1\}$ and $i, j \in N, i \neq j$; ties are nonreflexive and $l_{i i}=0$ for all $i$. We assume a two-sided link formation process [28], where a link can be created only with the mutual consent of both actors and severed by each actor unilaterally: a link $i j$ exists whenever $\min \left\{l_{i j}, l_{j i}\right\}=1$. The link decisions $l$ from $n$ actors define an $n \times n$ adjacency matrix $g$, where $g_{i j}=g_{j i}=\min \left\{l_{i j}, l_{j i}\right\}$. The matrix defines an undirected graph with the nodes representing the actors and the edges, the established interaction network. Let $N_{i} \equiv\left\{j \in N: g_{i j}=1\right\}$ denote the subset of the actors with whom $i$ interacts. These actors are $i$ 's neighbors or partners and their number equals $n_{i}=\left|N_{i}\right|$. Since social interactions usually involve time and effort, we define a linear cost function $\phi\left(n_{i}\right)=k n_{i}, k \geq 0$ for maintaining relations with $n_{i}$ neighbors.

If actors $i$ and $j$ are connected in $g$, they play the Chicken Game (also known as Hawk-Dove or Snowdrift Game). The name of the game refers to a form of a "duel" in which two drivers speed toward each other on a narrow road and the one who swerves to avoid the deadly collision is considered a coward, or a "chicken." The game situation also translates to the context of dividing a resource $[31,35]$ or more generally, bilateral bargaining or decision making [39, 45]. To capture the aspects of conflict and competition, we will here rename the actions to "Give" and "Take." The two actors may both decide to divide equally the benefits (Give, Give), or one of the actors may demand more (Take), while the other one acquiesces (Give). Yet, if both fail to yield (Take, Take), the result is a bargaining deadlock and a minimum payoff. In our version of the game, negotiation is costly. In other words, bargaining for equal division carries losses and, consequently, the dissimilar actions (Take, Give) and (Give, Take) generate the highest total benefit (Fig. 1). 


\begin{tabular}{c|c|c|} 
& Chicken & Dare \\
\hline Chicken & $c, c$ & $d, b$ \\
\hline Dare & $b, d$ & $e, e$ \\
\hline
\end{tabular}

\begin{tabular}{c|c|c} 
& Give & Take \\
\hline Give & 4,4 & 3,9 \\
\hline Take & 9,3 & 1,1 \\
\hline
\end{tabular}

Fig. 1. The underlying two-player Chicken Game in generalized form $(b>c>d>e ; b+d>2 c)$ and with actions and numerical payoffs as in the experiment.

Formally, we denote the common action set of the Chicken Game by $A=\{G, T\}$, representing Give and Take, and the payoff function by $\pi$. Actors can behave differently with different partners. Hence, we let $a_{i}=\left(a_{i 1}, \ldots, a_{i(i-1)}, a_{i(i+1)}, \ldots, a_{i n}\right)$ be the vector of action decisions of actor $i$, where $a_{i j} \in\{G, T\}$ and $i, j \in N, i \neq j$. Finally, we assume that an actor makes partner and action choices simultaneously. Thus, we model the strategic situation of the actor in the social game $\Gamma^{S}$ as a combination of her strategies in the network game and the Chicken Game: $s_{i}=\left(l_{i}, a_{i}\right)$, where $l_{i} \in\{0,1\}^{n-1}$ and $a_{i} \in\{G, T\}^{n-1}$. The actor's total payoff consists of the payoff she obtains from playing the underlying game with her neighbors (that is, with those with whom she has established a relation) minus the costs of maintaining her links. In formal terms, in the social game $\Gamma^{S}$, given the strategies of the other actors $s_{-i}=\left(s_{1}, \ldots, s_{i-1}, s_{i+1}, \ldots, s_{n}\right)$, the payoff of actor $i$ from playing some strategy $s_{i}=\left(l_{i}, a_{i}\right)$ is $\Pi_{i}\left(s_{i}, s_{-i}\right)=\sum_{j \in N_{i}} \pi\left(a_{i j}, a_{j i}\right)-k n_{i}$.

\subsection{Equilibria in the one-shot social game}

Since actors can discriminate in action choices among their partners, as a first crude approximation, we can view the multi-player social game as a collection of independent social games between all possible pairs in the group.

Hence, let us first consider the two-player one-shot social game with connection costs $k<d$ (as in the experiment). ${ }^{\text {a }}$ In a Nash equilibrium, each actor has selected the best response to the other actors' strategies and hence, no actor has an incentive to change strategy. Formally, a strategy profile $s^{*}=\left(s_{1}^{*}, \ldots, s_{n}^{*}\right)$ is a Nash equilibrium of the social game $\Gamma^{S}$ if $\forall i \in N$ and $\forall s_{i} \in\{0,1\}^{n-1} \times\{G, T\}^{n-1}$, $\Pi_{i}\left(s_{i}^{*}, s_{-i}^{*}\right) \geq \Pi_{i}\left(s_{i}, s_{-i}^{*}\right)$.

In our game, there are six pure-strategy Nash equilibria: in the first four, both actors choose not to have a link regardless of their action choices in the underlying game and in the other two, the pair establishes a link and coordinates on either (Take, Give) or (Give, Take). There is also a mixed-strategy Nash equilibrium in which each actor links and plays Give with probability $(d-e) /(b-c+d-e)$ and Take with the complementary probability.

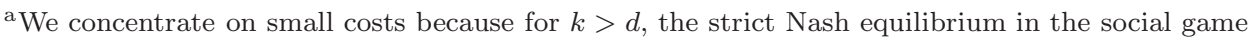
is trivial - the empty network. 


\begin{tabular}{c|c|c|c|c|} 
& $(0, G)$ & $(0, T)$ & $(1, G)$ & $(1, T)$ \\
\hline$(0, G)$ & $\mathbf{0 , 0}$ & $\mathbf{0 , 0}$ & 0,0 & 0,0 \\
\hline$(0, T)$ & $\mathbf{0 , 0}$ & $\mathbf{0 , 0}$ & 0,0 & 0,0 \\
\hline$(1, G)$ & 0,0 & 0,0 & $c-k, c-k$ & $\boldsymbol{d}-\boldsymbol{k}, \boldsymbol{b}-\boldsymbol{k}$ \\
\hline$(1, T)$ & 0,0 & 0,0 & $\boldsymbol{b}-\boldsymbol{k}, \boldsymbol{d}-\boldsymbol{k}$ & $e-k, e-k$ \\
\hline
\end{tabular}

Fig. 2. Pure-strategy equilibria in the one-shot two-player social game $(k<d)$. The Nash equilibria are shown in bold. The strict Nash equilibria are in the cells with thick borders.

We recognize that actors are unlikely to overlook straightforward opportunities to mutually increase their payoffs and coordinate on inefficient outcomes such as the empty network or the mixed-strategy equilibrium. Therefore, we will only concentrate on the strict Nash equilibria. A Nash equilibrium is strict if each actor gets a strictly higher payoff with her current strategy than she would with any other [18].

In the one-shot social game between two actors, the strict Nash equilibria are $(1, G),(1, T)$ and $(1, T),(1, G)$ (Fig. 2). ${ }^{\mathrm{b}}$ If an actor interacts with more than one other individual, we assume that her behavior choice toward one actor is independent from her behaviour choice toward any other. Then, in a social game between three or more actors, the strict Nash equilibria consist of the combinations of strict Nash equilibria in all possible dyads. Consequently, in the multi-player one-shot social game, the unique strict-Nash equilibrium interaction structure is the complete network and the action choices in each bilateral interaction are (Take, Give) or (Give, Take).

If we use directed edges to represent the payoff asymmetry in the Give-Take relation (pointing from the actor who has chosen Give to the actor who has chosen Take), we obtain a "tournament" - a directed graph in which each pair of nodes is connected by a single directed edge (Fig. 3). The equilibrium configurations can then be identified by the set of the indegrees of the nodes, which in our case is the distribution of Take actions. We will call this the tournament score. As in the experiment, we consider group sizes of three and five. In a three-node tournament, there are two different possible scores. In one score, all actors have an indegree of one: each actor "gives" to one actor and "takes" from one actor. In the other score, one actor has an indegree of one, another actor has an indegree of zero, and the third actor has an indegree of two. Similarly, in a five-node tournament, there are nine different possible scores [29]. Hence, based on the distribution of payoffs,

\footnotetext{
${ }^{\mathrm{b}}$ The other pure-strategy Nash equilibria are not strict because when an actor is in the no-link situation, she is indifferent between the two actions Take and Give. The mixed-strategy Nash equilibrium cannot be strict by definition [18].
} 
Three-person groups
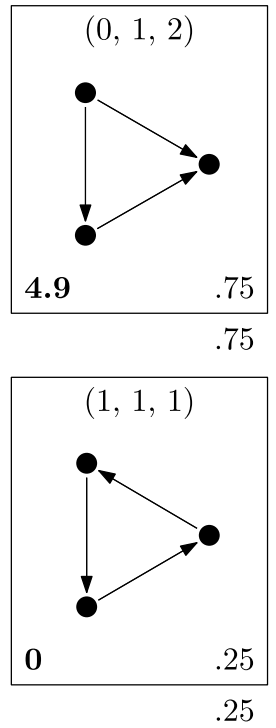

score

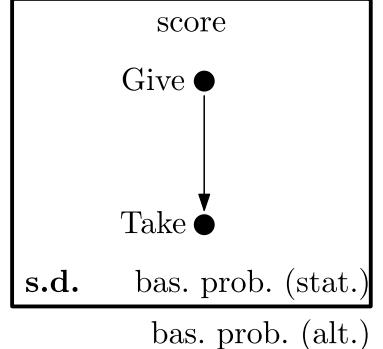

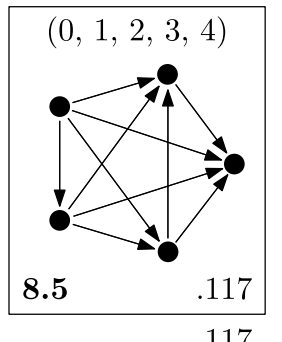

.117

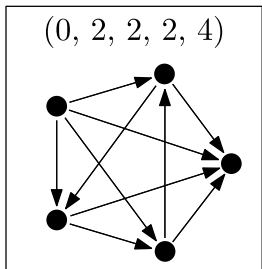

7.6

.039

.039

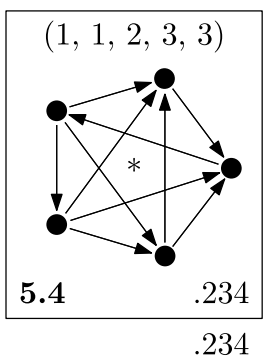

Five-person groups
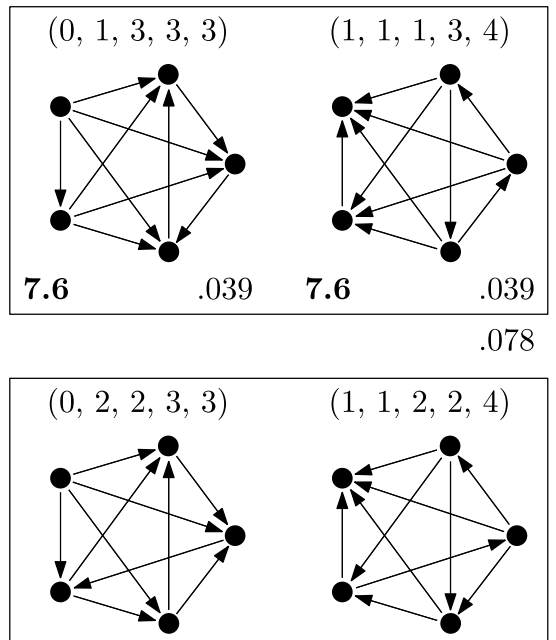

6.6

.117

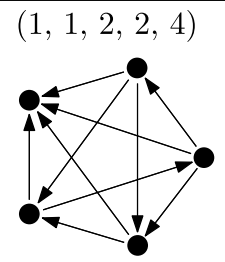

6.6

.117

.234

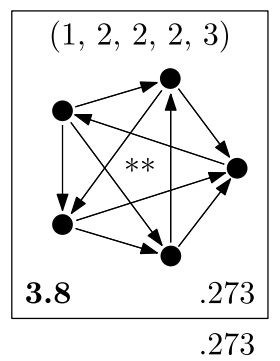

$(2,2,2,2,2)$

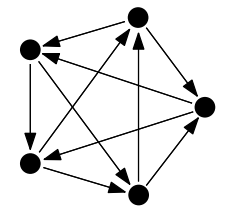

0

.023

.023

Fig. 3. The stationary and alternating conventions in the social game for three-person and fiveperson groups. There are two stationary and two alternating conventions in three-person groups and nine stationary and seven alternating conventions in five-person groups. Each cell illustrates the score (consisting of the number of Take actions per node), a graph of the tournament (each asterisk in the center of the graph indicates an additional nonisomorphic tournament for the same score), the distribution of payoffs (measured by the standard deviation in earnings for payoffs as in Fig. 1 and link costs of zero) and the baseline probability that the outcome occurs. Each graph represents a stationary convention, while each rectangular frame contains an alternating convention (for explanation, see last paragraph in Sec. 2.3). The key to reading the figure can be found in the lower left corner.

the number of possible strict-Nash equilibrium structures is two in the three-person social game and nine in the five-person social game (Fig. 3). ${ }^{\mathrm{c}}$

\footnotetext{
${ }^{\mathrm{c}} \mathrm{A}$ score does not uniquely determine a tournament. There are two nonisomorphic tournaments with a score of $(1,1,2,3,3)$ and three with $(1,2,2,2,3)$ (indicated on Fig. 3). As a result, the number of all nonisomorphic tournaments in a five-person group is twelve [34]. However, here we disregard the exact structure of the equilibrium networks.
} 


\subsection{Conventions in the repeated social game}

The strict Nash equilibria in the social game are both Pareto optimal ${ }^{\mathrm{d}}$ and welfare maximizing, although asymmetrical. If the actors play only once and make decisions simultaneously, without communication, and without the possibility to make binding commitments, it is unlikely that they coordinate on one of the equilibria. When the actors play the game repeatedly, however, they can resolve both the coordination and the asymmetry problems.

Repeated games allow for elaborate strategies based on reputation and retaliation. However, the multiplicity and complexity of these strategies often render the analytical solutions of dynamic games impractical. Hence, here we will concentrate only on two kinds of solutions based on simple two-period strategies: stationary conventions (actors play the same action in consecutive periods) or alternating conventions (actors alternate between two actions in consecutive periods).

When each actor chooses the same strict-Nash strategy in consecutive periods, the structures in Fig. 3 translate to stationary conventions. These stationary conventions can be ranked according to inequality in the distribution of payoffs. To do this, we can use the standard deviation in earnings. When the number of players is odd, there is only one completely egalitarian stationary convention (the structure with standard deviation $=0$ in Fig. 3). In this structure, each actor plays Take against half of her partners and Give against the other half.

Alternating conventions occur when each actor alternates between the two actions Take and Give in consecutive periods: i.e., for every $i \neq j, a_{i j(t+1)}=a_{j i(t)}$. This is equivalent to having the matrix of asymmetric action choices representing one of the strict-Nash equilibrium structures at time $t\left(\boldsymbol{A}_{t}\right)$ switch to its transpose the next period: $\boldsymbol{A}_{t+1}=\boldsymbol{A}_{t}^{T}$. For the two structures in the three-person groups and for five (out of nine) structures in the five-person groups, the transpose matrix is equivalent to the original. The remaining four structures for the five-person groups represent two self-inverse pairs, in the sense that the two action-choice matrices in each pair are each other's transpose. In sum, the number of possible egalitarian alternating conventions in terms of indegree distributions is two in three-person groups and seven in five-person groups.

\subsection{Baseline predictions}

Are certain convention structures more likely than others and in particular, are egalitarian convention structures more likely relative to the rest? To obtain a baseline prediction for the likelihood of each structure, we will assume that each relation in equilibrium is equally likely to be (Give, Take) or (Take, Give) and that this probability is independent among relations. Then, the number of possible ways to achieve a convention is eight in the three-person groups (equivalent to $2^{3}$, two options for

\footnotetext{
${ }^{\mathrm{d}}$ In a Pareto-optimal outcome, there is no actor whose utility can be improved without making another actor worse off.
} 
the direction of a relation for each of the three relations) and 1024 (or $2^{10}$ ) in the five-person groups. Of these, two and 24 in the three- and five-person groups, respectively, lead to an egalitarian stationary convention. e Consequently, our baseline expectation is that egalitarian stationary conventions emerge with a probability of $2 / 8=.25$ in three-person groups and with a probability of $24 / 1024=.023$ in five-person groups. If actors, however, have a preference for equality and manage to coordinate their actions, the egalitarian structures among the stationary conventions should be more common. The level of inequality as measured by the standard deviation of earnings and the baseline probability of occurring for each stationary and each alternating convention if directions in relations emerge independently are given in Fig. 3.

\section{Experimental Design}

To test the baseline predictions and see whether they are sensitive to some basic parameters (group size and link costs), we performed a computerized experiment. The advantage of this method is that it enables control over the environment. However, designing an environment that is simple and comprehensible, yet abstract enough to prevent any bias in subjects' responses poses a challenge. In our laboratory experiment, we addressed this issue by providing detailed instructions, practice rounds, straightforward utility functions and intuitive action choices, and a graphical representation of decision-relevant information.

The experiment was designed and conducted using the software program $z$ Tree [17]. Using the online recruitment system ORSEE [20], we invited 1964 subjects to participate in the study. A total of 165 subjects took part in one of the 11 sessions of 15 subjects each. Apart from four individuals, all subjects were students, primarily at Utrecht University and from a variety of disciplines: the most populous groups were from Economics or Business (33.9\%), the other Social Sciences (16.4\%), and Biology and Health Sciences (20\%). Subjects were between 17 and 44 years old (with an average age of 21.7 ), $58.2 \%$ female and $75.8 \%$ Dutch.

The 11 experimental sessions took place in November-December, 2009 in the Experimental Laboratory for Sociology and Economics (ELSE) at Utrecht University. At the beginning of the session, the 15 subjects were randomly assigned to a computer and given instructions on paper in either English or Dutch, according to their preference (a choice between the two languages was also available for the computer interface). The instructions explained in detail how the session proceeds, how the game is played and how one's earnings depend on one's own and the others' choices. Furthermore, the instructions informed each participant that the points they earn will be converted at the rate of one euro per 125 points at the end of the

\footnotetext{
eFor small groups, the probabilities of each outcome can be computed by estimating the number of matrices mapping on the ordered score set times the number of distinguishable permutations of the set [38]. This is equivalent to the number of ways of labeling the nodes for the particular score.
} 
experiment and that they will be paid in private. ${ }^{\mathrm{f}}$ After reading the instructions, the subjects played in four different treatments: two group sizes (groups of three or five participants), each in two cost conditions (with and without costs for maintaining relations). The order of the treatments was varied in as balanced a way as possible between sessions. This means that about the same number of subjects had first lower costs and then higher costs. Also, about the same number of subjects started with groups of size 3 and groups of size 5 . Still, subjects always finished the two cost conditions in the first group size before starting interaction in the other group size. In this way, we balanced learning effects over the conditions due to the within-subjects experimental design, while minimizing the confusion participants experience due to changing instructions. ${ }^{\mathrm{g}}$

Each of the four treatments was repeated twice (repetitions were called rounds), for a total of eight rounds. There were also two short unpaid practice rounds - one at the beginning of the session and the other before the treatments with a different group size. At the beginning of each round, subjects were randomly allocated to a group. The other participants were anonymous and could not be identified between rounds. In addition, no communication between subjects was allowed at any time during the experiment.

Each round consisted of 20 periods. In each period, subjects had to choose an action toward each other participant in the group. The other participants in the group could be identified by a randomly assigned number that stayed fixed throughout the 20 periods. The action choices were "No relation," "Take", or "Give." We chose these particular action names to give participants an intuitive understanding of the game. We deemed this necessary since the game involved a large number of decisions (640 per session). We realize that the positive/negative connotations of the action words might bias the behavior; however, the results do not suggest that this is the case (in particular, coordination on Give-Give was uncommon, as theoretically expected).

The payoffs from each interaction were as given in Fig. 2 with $b=9, c=4$, $d=3$, and $e=1$. In the condition with link costs, $k=2$, otherwise $k=0$.

In the first period of a round, subjects started from an empty network. In each period after the first one, subjects received information about the structure of the resulting network from the previous period, each participant's combined payoff and each partner's decision toward them (the latter, as long as the subject had not chosen "No relation"). The network was visualized in a graph where each type of relation was uniquely depicted by an arrow (see Fig. 4). This allowed subjects to extrapolate the decisions made in relations they were not involved in.

\footnotetext{
${ }^{f}$ The complete instructions are available from the authors upon request.

${ }^{g}$ Supplementary analyses not reported here reveal that a group is more likely to converge to a convention the more rounds the participants have played. However, the ordering of treatments (whether participants played in the smaller groups first or whether they played in the no-cost condition first) has no effect on their likelihood to coordinate on a convention. In other words, subjects indeed learn from playing the game but they do not learn better in any particular treatment.
} 


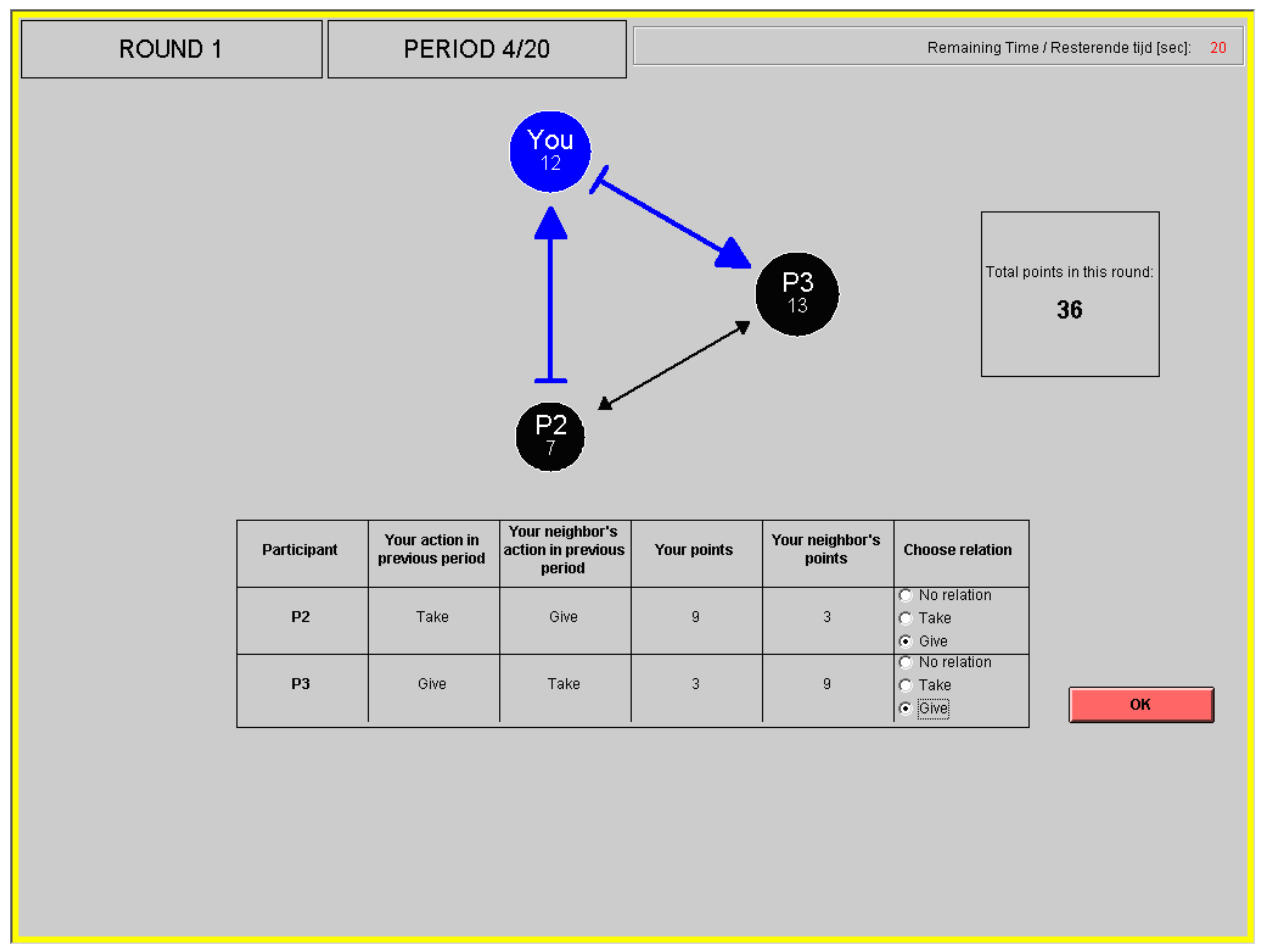

Fig. 4. Subject screen in the treatment with three-person groups and no link costs. (Note: Since the instructions explained the meaning of the different arrows, the current subject could extrapolate that P2 and P3 both chose Give toward each other.)

After playing the game, subjects were asked to complete a standard questionnaire covering background information and social preferences. Finally, subjects received their monetary earnings. Each session lasted approximately one hour and 45 minutes and subjects' earnings ranged from eight to 18 euros, with an average payment of 15 euros.

\section{Results}

The experimental data comprise observations of the realized network structure for each of 20 periods in 352 groups (11 sessions times four rounds of five three-person groups and four rounds of three five-person groups, for a total of 220 three-person and 132 five-person groups). We execute the analyses at the level of the group. We look at whether the group achieves an egalitarian convention, whether the established egalitarian convention is stationary, and at the particular structure of the established convention.

Similar to how we define a convention, we assume that a group reaches one if it coordinates on a strict Nash equilibrium for at least two consecutive periods. 
In a stationary convention, the group coordinates on the same strict Nash equilibrium and in an alternating convention, the group alternates between two strict Nash equilibria whose adjacency matrices are each other's transpose. ${ }^{\mathrm{h}}$ Additionally, we assume that there can be only one converged convention per group. If a group deviates from an already established convention to coordinate on another, we presume that this could be due to either boredom or purposive search. In the first case, the initial convention gets disrupted after it has lasted for too long and in the second case, it gets disrupted after a short period of time because at least one member would like to search for a different convention. In both cases, we expect the preferred convention to last longer. Thus, for the 12 groups in our experiment that reached more than one kind of convention, we selected the convention that was maintained for the longest time. In most of the cases (9 out of 12), this was also the last convention on which the group coordinated.

Overall, 182 of the 352 groups $(51.7 \%)$ converged to a convention. All of the conventions in the five-person groups (43 out of 43) and almost all of the conventions in the three-person groups (135 out of 139) were egalitarian (Table 1). These numbers show that, compared to egalitarian conventions, inegalitarian conventions are much less likely to be stable. Furthermore, the four inegalitarian conventions that did stabilize occurred between three and six times during the particular group interaction but never lasted more than two consecutive periods. In comparison, egalitarian conventions were on average maintained for 11.0 consecutive periods (s.d. $=6.1)$.

Although unaffected by link costs, convergence to an egalitarian convention was almost twice as common in the three-person groups compared to the five-person groups (61.4\% versus $32.6 \%$, respectively). Most significantly, almost all of the egalitarian conventions were alternating. The only stationary egalitarian convention occurred in a three-person group in the condition with costs for links (Table 1).

Do the particular structures of alternating egalitarian conventions emerge according to the baseline prediction? Table 2 allows us to compare the baseline and the observed probabilities for each structure. On the one hand, we notice that some structures appeared more often than expected: $(0,1,2),(0,2,2,2,4)$, and $(0$, $1,2,3,4)$. In particular, the latter alternating-convention structure occurred more than $50 \%$ of the time although the expected frequency was $11.7 \%$. On the other hand, no groups managed to coordinate on the structures $(0,1,3,3,3)$ and $(2,2$, $2,2,2)$ but this might be due to their extremely small predicted probabilities (.078 and .023, respectively) combined with the small number of instances of successful coordination (only 43 cases). We tested whether the deviations from the baseline probabilities are significant using a conditional logit model that controlled for the

\footnotetext{
${ }^{\mathrm{h}}$ We repeated the analyses with a stricter operationalization of convergence by defining stationary conventions as at least three consecutive periods of identical equilibria and alternating conventions as at least four periods of alternation between two equilibria. The number of groups that converged was smaller (129 compared to 182) but qualitatively, the results were the same.
} 
Table 1. Proportion of groups that converged to inegalitarian conventions, proportion of groups that converged to egalitarian conventions, and proportion of alternating conventions among groups that converged to egalitarian conventions by group size and cost condition. Numbers in brackets show true counts.

\begin{tabular}{|c|c|c|c|}
\hline & \multicolumn{2}{|c|}{ Conventions } & \multirow{2}{*}{$\begin{array}{l}\text { Egalitarian } \\
\text { Alternating }\end{array}$} \\
\hline & Inegalitarian & Egalitarian & \\
\hline \multicolumn{4}{|c|}{ Three-person groups } \\
\hline No link costs & $0.036(4 / 110)$ & $0.609(67 / 110)$ & $1.000(67 / 67)$ \\
\hline Link costs & & $0.618(68 / 110)$ & $0.985(67 / 68)$ \\
\hline Total & $0.018(4 / 220)$ & $0.614(135 / 220)$ & $0.993(134 / 135)$ \\
\hline \multicolumn{4}{|c|}{ Five-person groups } \\
\hline No link costs & & $0.348(23 / 66)$ & $1.000(23 / 23)$ \\
\hline Link costs & & $0.303(20 / 66)$ & $1.000(20 / 20)$ \\
\hline Total & & $0.326(43 / 132)$ & $1.000(43 / 43)$ \\
\hline Total & $0.011(4 / 352)$ & $0.506(178 / 352)$ & $0.994(177 / 178)$ \\
\hline
\end{tabular}

Table 2. Baseline and observed proportions of alternating-convention structures. Numbers in brackets show true counts.

\begin{tabular}{|c|c|c|c|c|}
\hline & \multicolumn{2}{|c|}{ Three-person groups } & \multicolumn{2}{|c|}{ Five-person groups } \\
\hline & Bas. & Obs. & Bas. & Obs. \\
\hline$(0,1,2)$ & 0.750 & $0.858(115 / 134)$ & & \\
\hline$(1,1,1)$ & 0.250 & $0.142(19 / 134)$ & & \\
\hline$(0,1,2,3,4)$ & & & 0.117 & $0.512(22 / 43)$ \\
\hline$(0,1,3,3,3) /(1,1,1,3,4)$ & & & 0.078 & $0.000(0 / 43)$ \\
\hline$(0,2,2,2,4)$ & & & 0.039 & $0.116(5 / 43)$ \\
\hline$(0,2,2,3,3) /(1,1,2,2,4)$ & & & 0.234 & $0.163(7 / 43)$ \\
\hline$(1,1,2,3,3)$ & & & 0.234 & $0.070(3 / 43)$ \\
\hline$(1,2,2,2,3)$ & & & 0.273 & $0.140(6 / 43)$ \\
\hline$(2,2,2,2,2)$ & & & 0.023 & $0.000(0 / 43)$ \\
\hline
\end{tabular}

interdependence between observations due to the fact that the same subjects were involved in multiple groups. This analysis showed that mainly the structures $(0$, $1,2)$ and $(0,1,2,3,4)$ occur more often than expected (for technical details, see Ref. 48).

In sum, we found that certain egalitarian alternating conventions are more likely to occur than we would expect based on chance. Paradoxically, the most common structures are the ones with the most inegalitarian distribution of payoffs at any single period (Fig. 5). We uncovered two related individual-level mechanisms behind this phenomenon. On the one hand, the outcomes tended to have extreme action distributions because subjects randomized their action choices quite poorly in the first period: they tended to choose the same action in all of their relations much more 

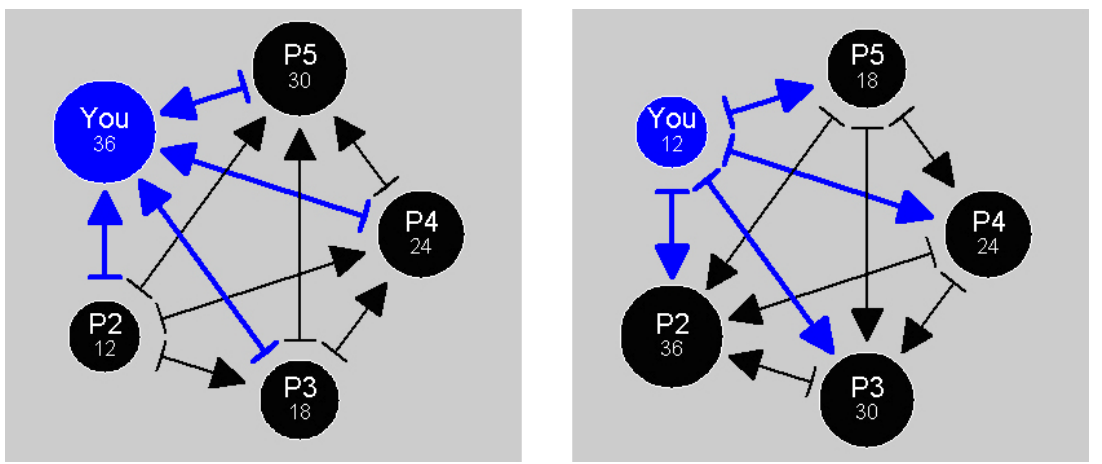

Fig. 5. Visualizations of the $(0,1,2,3,4)$ alternating convention in two consecutive periods, taken form subject screens in the treatment with five-person groups and no link costs.

often than any other possible combination of actions. Because of the bias in initial strategies, it sometimes happened that more than two subjects would start with allTake or all-Give actions. But then, instead of adjusting their strategy accordingly, those subjects tended to insist on their positions. It often took at least three periods before someone "retreated." It appears that being in an all-Take/all-Give position had some inherent attractiveness (one possibility is that it decreases the cognitive demands when playing the game). Thus, on the other hand, the hierarchization of the action configurations in the convention structures was the result of subjects strategically seeking particular structural positions.

\section{Discussion and Conclusions}

In this paper, we used a model of the Chicken Game with endogenous partner selection and partner-specific action choices to demonstrate that groups of individuals involved in asymmetric dyadic relations can achieve egalitarian outcomes by coordinating on conventions based on direct or indirect reciprocity. Data obtained from a computerized laboratory experiment revealed that, in reality, only the egalitarian conventions are stable: they occur remarkably more often and last significantly longer than any inegalitarian conventions. This observation matches previous results from network experiments, which have shown that efficient but inegalitarian equilibria are unlikely to stabilize in an anonymous experimental setting [15].

Interestingly, the egalitarian alternating conventions are much more likely to emerge than the egalitarian stationary conventions (there was only a single instance of a group achieving a stationary convention). We can think of three explanations for this result. On the one hand, coordination between three or more people is more difficult to achieve than coordination between two. On the other hand, direct reciprocity ensures equality in each dyad and for people, relationship-level outcomes might take precedence over group-level outcomes. Finally, we also admit that this result might have been influenced by the experimental setup: the fact that individuals interacted in discrete time and that they had to make a choice of action 
in every period might have steered them toward alternating equilibria. In contrast, continuous time provides less clear periodicity and more continuity in action choices. Although experiments with continuous time do not preclude "taking turns" type of behavior $[4,5]$, they might be more conductive to stationary equilibria.

In general, our finding that indirect reciprocity is an unlikely outcome aligns well with previous theoretical and experimental results. First, evolutionary biology models show that indirect reciprocity is less likely to evolve in a population than direct reciprocity $[8,36]$. Second, generalized-exchange experiments reveal that when the interaction structure is fixed and circular (i.e., relatively simple), indirect reciprocity becomes less likely to stabilize as the group size increases [21, 32, 51]. Our experiment shows that indirect reciprocity is even less likely when the interaction structure is adaptive and direct reciprocation is possible. Yet, large-scale interaction structures based on indirect reciprocity have been observed in natural social settings: prominent examples are the Kula trading ring among South Pacific islanders [30] and the kinship relations among aboriginal tribes [3]. This suggests that indirect reciprocity may be viable only when direct reciprocity is precluded (as in incestuous relations [3]) or when the interaction structure is strongly constrained (for example, due to geography [52]). Future research should explore whether these or other particular conditions foster conventions based on indirect reciprocity.

Since our study relied on an analytic theoretical approach and an experimental empirical test, the conclusions drawn here are limited to very small groups. One particularly promising direction for future research is to investigate the structure and dynamics of reciprocity chains and cycles in large-scale social networks. This endeavor will require agent-based computational models to generate predictions and longitudinal data on complete large-scale networks, today relatively easily obtainable from the Web.

\section{Acknowledgments}

This project is part of the Utrecht University High Potential program "Dynamics of Cooperation, Networks, and Institutions" of Vincent Buskens and Stephanie Rosenkranz. We thank Vincenz Frey for assistance in carrying out the experiments, Jeroen Weesie for help with the analyses, and Anne-Rigt Poortman and Ineke Maas for useful comments and suggestions.

\section{References}

[1] Axelrod, R., The Evolution of Cooperation (Basic Books, New York, 1984).

[2] Axelrod, R. and Hamilton, W. D., The evolution of cooperation, Science 211 (1981) 1390-1396.

[3] Bearman, P., Generalized exchange, Am. J. Sociol. 102 (1997) 1383-1415.

[4] Berninghaus, S. K., Ehrhart, K. M. and Ott, M., A network experiment in continuous time: the influence of link costs, Exp. Econ. 9 (2006) 237-251. 
[5] Berninghaus, S. K., Ehrhart, K. M. and Ott, M., Myopically forward-looking agents in a network formation game: Theory and experimental evidence (2008), http://www. sfb504.uni-mannheim.de/publications/dp08-02.pdf.

[6] Berninghaus, S. K. and Vogt, B., Network formation and coordination games (2003), http://www.sfb504.uni-mannheim.de/publications/dp03-16.pdf.

[7] Bornstein, G., Budescu, D. and Zamir, S., Cooperation in intergroup, N-person and two-person games of Chicken, J. Confl. Resolut. 41 (1997) 384-406.

[8] Boyd, R. and Richerson, P. J., The evolution of indirect reciprocity, Soc. Netw. 11 (1989) 213-236.

[9] Bramoullé, Y., López-Pintado, D., Goyal, S. and Vega-Redondo, F., Network formation and anti-coordination games, Int. J. Game Theory 33 (2004) 1-19.

[10] Buskens, V., Corten, R. and Weesie, J., Consent or conflict: Coevolution of coordination and networks, J. Peace Res. 45 (2008) 205-222.

[11] Corbae, D. and Duffy, J., Experiments with network formation, Games Econ. Behav. 64 (2008) 81-120.

[12] Corten, R. and Buskens, V., Co-evolution of conventions and networks: An experimental study, Soc. Netw. 32 (2010) 4-15.

[13] Corten, R. and Cook, K. S., Cooperation and reputation in dynamic networks, in Proceedings of the First International Conference on Reputation: Theory and Technology - ICORE 09, ed. Poalucci, M. (Gargonza, Italy, 2009).

[14] Eguíluz, V. M., Zimmermann, M. G., San Miguel, M. and Cela-Conde, C. J., Cooperation and the emergence of role differentiation in the dynamics of social networks, Am. J. Sociol. 110 (2005) 977-1008.

[15] Falk, A. and Kosfeld, M., It's all about connections: Evidence on network formation (2003), http://papers.ssrn.com/sol3/papers.cfm?abstract_id=390020.

[16] Fehl, K., van der Post, D. J. and Semmann, D., Co-evolution of behaviour and social network structure promotes human cooperation, Ecol. Lett. 14 (2011) 546-551.

[17] Fischbacher, U., Z-tree: Zurich toolbox for ready-made economic experiments, Exp. Econ. 10 (2007) 171-178.

[18] Fudenberg, D., Kreps, D. M. and Levine, D. K., On the robustness of equilibrium refinements, J. Econ. Theory 44 (1988) 354-380.

[19] Goyal, S. and Vega-Redondo, F., Network formation and social coordination, Games Econ. Behav. 50 (2005) 178-207.

[20] Greiner, B., The online recruitment system ORSEE 2.0 - a guide for the organization of experiments in economics, http://orsee.sourceforge.net/web/files/ orsee_pub.pdf (2004).

[21] Greiner, B. and Levati, M. V., Indirect reciprocity in cyclical networks: An experimental study, J. Econ. Psychol. 26 (2005) 711-731.

[22] Gross, T. and Blasius, B., Adaptive coevolutionary networks: A review, J. Roy. Soc. Interface 5 (2008) 259-271.

[23] Hanaki, N., Peterhansl, A., Dodds, P. S. and Watts, D. J., Cooperation in evolving social networks, Manag. Sci. 53 (2007) 1036-1050.

[24] Hauk, E., Multiple Prisoner's Dilemma games with(out) an outside option: An experimental study, Theory Decis. 54 (2003) 207.

[25] Hauk, E. and Nagel, R., Choice of partners in multiple two-person Prisoner's Dilemma games, J. Confl. Resolut. 45 (2001) 770-793.

[26] Hojman, D. A. and Szeidl, A., Endogenous networks, social games and evolution, Games Econ. Behav. 55 (2006) 112-130.

[27] Jackson, M. O. and Watts, A., On the formation of interaction networks in social coordination games, Games Econ. Behav. 41 (2002) 265-291. 
[28] Jackson, M. O. and Wolinsky, A., A strategic model of social and economic networks, J. Econ. Theory 71 (1996) 44-74.

[29] Landau, H., On dominance relations and the structure of animal societies: III. The condition for a score structure, Bull. Math. Biol. 15 (1953) 143-148.

[30] Malinowski, B., 51. Kula; the circulating exchange of valuables in the archipelagoes of Eastern New Guinea, Man 20 (1920) 97-105.

[31] Maynard Smith, J. and Price, G. R., The logic of animal conflict, Nature 246 (1973) $15-18$.

[32] Molm, L. D., Collett, J. L. and Schaefer, D. R., Building solidarity through generalized exchange: A theory of reciprocity, Am. J. Sociol. 113 (2007) 205-242.

[33] Molm, L. D., The structure of reciprocity, Soc. Psychol. Quart. 73 (2010) 119-131.

[34] Moon, J. W., Topics on Tournaments (Holt, New York, 1968).

[35] Neugebauer, T., Poulsen, A. and Schram, A., Fairness and reciprocity in the HawkDove game, J. Econ. Behav. Organ. 66 (2008) 243-250.

[36] Nowak, M. A. and Sigmund, K., Evolution of indirect reciprocity, Nature 437 (2005) 1291-1298.

[37] Pfeiffer, T., Rutte, C., Killingback, T., Taborsky, M. and Bonhoeffer, S., Evolution of cooperation by generalized reciprocity, Proc. R. Soc. B 272 (2005) 1115-1120.

[38] Rapoport, A., Outline of a probabilistic approach to animal sociology: I, Bull. Math. Biol. 11 (1949) 183-196.

[39] Rapoport, A. and Chammah, A. M., The game of Chicken, Am. Behav. Sci. 10 (1966) 10-14, 23-28.

[40] Rapoport, A., Guyer, M. and Gordon, D. G., The 2 X 2 Game (University of Michigan Press, Ann Arbor, 1976).

[41] Riedl, A. and Ule, A., Exclusion and cooperation in social network experiments (2002), http://cess.nyu.edu/UleRiedl.pdf.

[42] Roca, C. P., Cuesta, J. A. and Sánchez, A., Evolutionary game theory: Temporal and spatial effects beyond replicator dynamics. Phys. Life Rev. 6 (2009) 208-249.

[43] Schuessler, R., Exit threats and cooperation under anonymity, J. Confl. Resolut. 33 (1989) 728-749.

[44] Skyrms, B. and Pemantle, R., A dynamic model of social network formation, Proc. Nat. Acad. Sci. 97 (2000) 9340-9346.

[45] Snyder, G. H., 'Prisoner's Dilemma' and 'Chicken' models in international politics, Int. Stud. Quart. 15 (1971) 66-103.

[46] Szabó, G. and Fáth, G., Evolutionary games on graphs, Phys. Rep. 446 (2007) 97-216.

[47] Taylor, M., The Possibility of Cooperation. Revised. (Cambridge University Press, Cambridge, 1987).

[48] Tsvetkova, M., Egalitarian Networks from Asymmetric Relations: Coordination on Reciprocity in a Social Game of Hawk-Dove, M.S. thesis, Utrecht University, Utrecht, Netherlands (2010).

[49] Vanberg, V. J. and Congleton, R. D., Rationality, morality and exit, Am. Polit. Sci. Rev. 86 (1992) 418-431.

[50] Wang, J., Suri, S. and Watts, D. J., Cooperation and assortativity with dynamic partner updating. Proc. Nat. Acad. Sci. 109 (2012) 14363-14368.

[51] Yamagishi, T. and Cook, K. S., Generalized exchange and social dilemmas, Soc. Psychol. Quart. 56 (1993) 235-248.

[52] Ziegler, Rolf, What makes the Kula go round?: A simulation model of the spontaneous emergence of a ceremonial exchange system, Social Networks 30 (2008) 107-126. 